\title{
Evaluation of insulin resistance in acromegalic patients before and after treatment with somatostatin analogues
}

\author{
C.L. Ronchi, E. Orsi, C. Giavoli, V. Cappiello, P. Epaminonda, P. Beck-Peccoz, and M. Arosio \\ Institute of Endocrine Sciences, University of Milan, Ospedale Maggiore IRCCS, Milan, Italy
}

\begin{abstract}
Many studies have recently shown that simple computer-solved indices, based on fasting glucose and insulin levels, closely mirror the euglycemic clamp technique in studying insulin resistance or pancreatic insulin secretion. Few data are at present available on the evaluation of these novel indices in acromegalic patients, known to be $\mathrm{GH}$-dependent insulin-resistant subjects, in particular during medical treatment with somatostatin analogues. Indeed, these drugs are able to inhibit not only GH and IGF-I levels, but also insulin and glucagon pancreatic secretion, with contrasting effects on glucose metabolism. In this study, insulin resistance was evaluated by the homeostasis model assessment (HOMA-IR) and insulin sensitivity by quantitative insulin check index (QUICKI) in 27 normoglycemic acromegalic patients, before and after 6-month therapy with somatostatin analogues (lanreotide-SR $30-60 \mathrm{mg} \mathrm{ev}$ ery 7-28 days in 15 and octreotide-LAR $20-30 \mathrm{mg}$ every 28 days in 12). Thirty-five age- and sexmatched healthy subjects and 17 surgically treat-
\end{abstract}

\section{INTRODUCTION}

It is known that about $50 \%$ of acromegalic patients are affected by impaired glucose tolerance (IGT) and that $10-25 \%$ have overt Type 2 diabetes mellitus (1). Elevated insulin levels and reduced insulin sensitivity $(2,3)$ are often present also in normoglycemic patients. Insulin resistance, probably the most important $\mathrm{GH}$-dependent diabetogenic effect, is known to be an important cardiovascular risk factor and could play a role in the increased morbidity and mortality of acromegalic patients.

Key-words: Acromegaly, glucose metabolism, insulin resistance, somatostatin analogues.

Correspondence: Dr. Cristina L. Ronchi, Istituto di Scienze Endocrine, Pad. Granelli, Ospedale Maggiore IRCCS, Via F. Sforza, 35 - 20122 Milano, Italy. E-mail: cristina.ronchi@unimi.it

Accepted January 27, 2003. ed acromegalic patients (5 cured and 12 not cured) were studied as control groups. Before medical treatment, HOMA-IR was higher in acromegalic patients than in healthy controls $(4 \pm 3$ vs $1.7 \pm 0.7, p<0.05)$, while QUICKI was lower $(0.33 \pm 0.04$ vs $0.36 \pm 0.03, p<0.05)$. During medical therapy, HOMA-IR decreased to $2.4 \pm 1.6$ $(p<0.05)$ and became similar to that recorded in both healthy subjects and surgically treated patients. However, fasting glucose was increased and fasting insulin was decreased. QUICKI did not significantly change from basal values. No differences were observed between patients who normalized or not hormonal levels. The effects of the 2 drugs, though higher glucose levels were seen in patients treated with octreotide-LAR. In conclusion, this study demonstrates that medical treatment is able to improve insulin resistance, even if only successful surgery is able to completely normalize both HOMA-IR and QUICKI.

(J. Endocrinol. Invest. 26: 533-538, 2003)

${ }^{\circ}$ 2003, Editrice Kurtis

As a possible patho-physiological mechanism, either a receptor (4) or post-receptor defect (5), responsible for an alteration in non-oxidative glucose metabolism (6), may be involved. These defects cause a reduced suppression of hepatic glucose production and an impaired peripheral glucose uptake (4). An additional mechanism involved in the hepatic and peripheral reduced insulin sensitivity is the GH-dependent excess of circulating free fatty acid levels (7). Recently, a large number of simple computer-solved indices, based on fasting plasma glucose and insulin concentrations, in particular the homeostasis model assessment (HOMA) (8-10) and the quantitative insulin check index (QUICKI) (11), have been proposed to estimate or predict the degree of insulin resistance (IR) or the $\beta$ cell function in vivo. Many Authors demonstrated a good correlation between the values of HOMA-IR and QUICKI (12-14) and those obtained by the "gold standard" of insulin sensitivity, i.e. hyperinsulinemic euglycemic glucose clamp (15). 
Therefore, these novel indices have been employed in order to evaluate glucose tolerance status in the general population (16), as well as in typically insulinresistant subjects, such as those with Type 2 diabetes $(17,18)$, obesity (19), blood hypertension (20) and polycystic ovarian syndrome (21). Moreover, it has also been demonstrated that HOMA-IR may be used as an independent predictor of cardiovascular disease in Type 2 diabetes $(22,23)$. Finally, recent studies showed that QUICKI better correlates with the euglycemic clamp than both HOMA-IR and minimal model analysis of a frequently sampled iv glucose tolerance test, and it seems also the preferable method to be employed in large-scale and epidemiological studies (24). Insulin sensitivity and $\beta$ cell function were investigated by HOMA in normoand hyperglycemic acromegalic patients, before and after successful surgical treatment (25), but data on the use of HOMA-IR and/or QUICKI to monitor the effects on glucose metabolism of the 2 depot somatostatin analogues, i.e. lanreotide slow release (lan-SR) or octreotide long-acting release (oct-LAR), are still lacking. Both formulations are able to inhibit in a similar manner pancreatic insulin and glucagon secretion, as well as $\mathrm{GH}$ secretion, in acromegalic patients (26-29). These metabolic effects are responsible for complex results on overall glycemic control, mainly depending on the balance between the improvement of $\mathrm{GH}$-dependent insulin resistance and the suppression of insulin and glucagon secretion.

The aim of this study was to evaluate carbohydrate metabolism and insulin sensitivity in acromegalic patients by using HOMA-IR and QUICKI, as well as to investigate the effects of medical therapy with 2 different somatostatin analogues on these parameters and to compare them with the results obtained in healthy subjects and surgically treated acromegalics.

\section{PATIENTS AND METHODS}

\section{Patients and treatment protocol}

A group of 27 acromegalic patients with active disease (16 F and $11 \mathrm{M}$, age: $43 \pm 12 \mathrm{yr}$, range: $22-66$; pre-medical treatment $\mathrm{GH}: 9.5 \pm 8.5 \mu \mathrm{g} / \mathrm{l}$, range: 2.5-25.5; IGF-I: $90 \pm 36 \mathrm{nmol} / \mathrm{l}$, range: $38-190)$ was studied. Mean duration of the disease before diagnosis was 6 \pm 4 yr (range: 2-15 yr). Twenty-four of these patients previously underwent unsuccessful surgical treatment and 13 underwent radiotherapy (7 patients by conventional therapy and 6 by gamma-knife). Two of the 27 patients had impaired glucose tolerance (IGT) before the beginning of the study, while none had Type 2 diabetes mellitus; 8 patients had hypertension.
Serum GH and IGF-I levels, fasting glucose and insulin levels, HOMA-IR and insulin sensitivity by QUICKI, were evaluated before and after a period of 6 months of medical treatment with long-acting somatostatin analogues.

In particular, 15 of the 27 patients (10 $\mathrm{F}$ and $5 \mathrm{M}$ ) were treated with lan-SR at the variable dose of 30 $\mathrm{mg}$ im every 7-14 days or $60 \mathrm{mg}$ im every $21-28$ days and 12 patients ( $6 \mathrm{~F}$ and $6 \mathrm{M}$ ) with oct-LAR at the dose of 20-30 mg im every 28 days.

Moreover, in a subgroup of 12 acromegalic patients (8 $\mathrm{F}$ and $4 \mathrm{M}, 5$ treated with lan-SR and 7 with oct$L A R$ ) all the results were evaluated also after a mean period of 24-month therapy.

Thirty-five sex- and age-matched healthy subjects and 17 normo-glycaemic acromegalic patients $(12 \mathrm{~F}$ and $5 \mathrm{M}$; age: $39 \pm 10 \mathrm{yr}$ ), evaluated after transsphenoidal surgery ( 5 cured and 12 not cured), were studied as control groups. Criteria for cure of acromegaly were normalization of IGF-I levels, adjusted for sex and age, and $\mathrm{GH}$ levels below $2.5 \mu \mathrm{g} / \mathrm{l}$.

The Local Ethics Committee had approved the protocol study and all the patients gave their informed written consent to participate in this study.

\section{Hormone assays}

Serum GH levels were measured by an immunofluorimetric assay method (AutoDelfia kit) supplied by, Wallac OY, Turku, Finland. The sensitivity is $0.01 \mu \mathrm{g} / \mathrm{l}$, the intra- and inter-assay coefficients of variation are 2 and $1.7 \%$, respectively. Serum IGF-I was measured by a radioimmunoassay with a commercial kit supplied by Mediagnost, Tübingen, Germany, able to measure total IGF-I by separating IGF-I from IGFBP by acidification in IGF-II excess. IGF-II cross-reactivity is less than $0.05 \%$; the intra- and interassay coefficients of variation are 3.2 and $7.4 \%$, respectively. The normal values range from 15 to $44 \mathrm{nmol} / \mathrm{l}$ at the age of 20-30 yr, 14 to $42 \mathrm{nmol} / \mathrm{l}$ at 30-40 yr, 13 to $40 \mathrm{nmol} / \mathrm{l}$ at $40-50 \mathrm{yr}, 12.6$ to $38.2 \mathrm{nmol} / \mathrm{l}$ at $50-60$ $\mathrm{yr}$ and 12 to $37 \mathrm{nmol} / \mathrm{l}$ at $60-70 \mathrm{yr}$.

Plasma glucose was measured with standard methods (enzymatic method, intra- and interassay coefficients of variation are 2.1 and $2.6 \%$, respectively). Serum insulin concentrations were assessed by an immuno-enzymatic one-step assay (Medgenics Diagnostics, Belgium); its sensitivity is $0.15 \mathrm{mU} / \mathrm{l}$; intra- and inter-assay coefficients of variation are 4 and $7.5 \%$, respectively. Insulin resistance was determined using HOMA-IR and QUICKI methods. The former is a computer-solved model based on fasting serum insulin (FI) and plasma glucose concentrations (FG): HOMA-IR=FI (mU/l) x FG (mmol/l)/22.5 (8). The QUICKI is a novel index used for the evaluation of insulin sensitivity, derived from fasting data with var- 
ious transformations and ultimately defined in the following formula: QUICKI=1/[log FG (mg/dl)+log $\mathrm{FI}(\mathrm{mU} / \mathrm{l})](11)$.

\section{Statistical analyses}

All results were expressed as the mean $\pm \mathrm{SD}$. The comparisons between the results obtained before and after medical therapy in the same group of patients were performed using paired Student's $t$ test. The comparisons between acromegalic patients and normal subjects were performed using nonparametric test. Correlation between different parameters was evaluated by linear regression analysis. Chi-square test was used to compare percentage of patients with values out of normal range before and after therapy. Values of $p<0.05$ were considered statistically significant.

\section{RESULTS}

Before starting medical therapy, acromegalic patients had mean fasting insulin levels higher than healthy subjects $(p<0.05)$, with values above 14.3 $\mathrm{mU} / \mathrm{l}$ (mean+2 SD of healthy subjects) in 15 of 27 patients. They also had HOMA-IR higher than controls $(4 \pm 3$ vs $1.7 \pm 0.7, p<0.05)$, with values above 3.1 (mean+2 SD of healthy subjects) in 14 patients. They had QUICKI lower than healthy subjects $(0.33 \pm 0.04$ vs $0.36 \pm 0.03, p<0.05)$, with values lower than 0.30 (mean-2 SD of healthy subjects) in 9 patients. No correlation was found between basal HOMA-IR or QUICKI and serum GH levels, IGF-I levels or duration of disease.

After 6 months of medical therapy, GH levels significantly decreased, reaching values below $2.5 \mu \mathrm{g} / \mathrm{l}$ in 13 of 27 patients, while IGF-I levels normalized in 10 patients (Table 1). Fasting glucose levels significantly increased compared to baseline, while fasting insulin levels significantly decreased, remaining higher than $14.3 \mathrm{mU} / \mathrm{l}$ only in 6 patients ( $p<0.05 \mathrm{vs}$ basal by $\chi$-square test) and becoming similar to those of healthy subjects and surgically treated patients. As a consequence of these modifications in glucose and insulin levels, an overall decrease of HOMA-IR values (to $2.4 \pm 1.6, p<0.05$ vs basal), which became similar to those of other control groups, was observed (Fig. 1). QUICKI values were stable during medical treatment and still lower than healthy controls, but similar to surgically treated patients (Fig. 1). HOMA-IR were normal in 20 and QUICKI in 24 of 27 patients during medical therapy (both $p<0.05$ vs basal by $\chi$-square test), while the 2 indices normalized in 6 of the 12 surgically not cured patients and in all the 5 surgically cured patients ( $p<0.05$ vs basal by $\chi$-square test). Both
Table 1 - Hormonal and biochemical parameters in acromegalic patients before and after 6-month medical therapy: comparison with surgically treated patients and healthy subjects.

\begin{tabular}{lcccc}
\hline & Basal & $\begin{array}{c}\text { Acro medical } \\
\text { therapy }\end{array}$ & $\begin{array}{c}\text { Acro } \\
\text { surgery }\end{array}$ & $\begin{array}{c}\text { Healthy } \\
\text { subjects }\end{array}$ \\
\hline $\begin{array}{l}\text { Age }(\mathrm{yr}) \\
\mathrm{GH}\end{array}$ & $\begin{array}{c}(\mathrm{no}=27) \\
43 \pm 12\end{array}$ & $\begin{array}{c}(\mathrm{no} .=27) \\
(\mathrm{no}=17)\end{array}$ & $\begin{array}{c}(\mathrm{no} .=35) \\
39 \pm 10\end{array}$ & $38 \pm 12$ \\
$\begin{array}{l}(\mu \mathrm{g} / \mathrm{l}) \\
\text { IGF-I } \\
(\mathrm{nmol} / \mathrm{l})\end{array}$ & $90 \pm 36$ & $2.7 \pm 2^{*}$ & $7.7 \pm 5.0$ & - \\
$\begin{array}{l}\mathrm{Glucose} \\
(\mathrm{nm} / \mathrm{l})\end{array}$ & $4.9 \pm 0.9$ & $5.4 \pm 0.8^{*}$ & $4.8 \pm 0.9$ & $4.7 \pm 0.3$ \\
$\begin{array}{l}\text { Insulin } \\
(\mathrm{mU} / \mathrm{l})\end{array}$ & $18 \pm 12^{\circ}$ & $10 \pm 6^{*}$ & $14 \pm 12$ & $8.3 \pm 3$ \\
\hline
\end{tabular}

${ }^{\circ} p<0.05$ vs healthy subjects.

${ }^{*} p<0.05$ vs baseline.
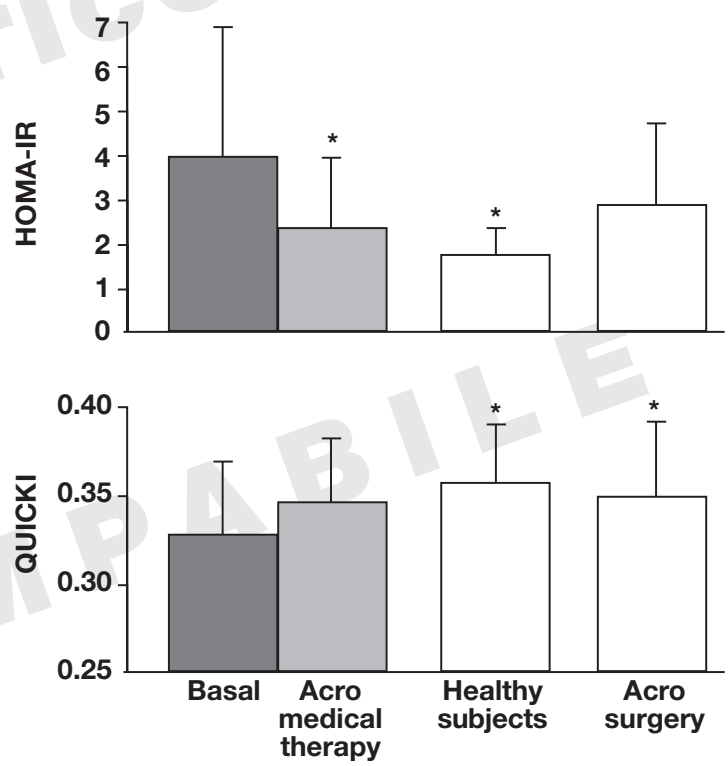

Fig. 1 - Insulin resistance by homeostasis model assessment (HOMA-IR) and insulin sensitivity by quantitative insulin check index (QUICKI) in 27 acromegalic patients before and after 6month medical therapy, in 35 healthy subjects and in 17 surgically treated patients. ${ }^{*} \mathrm{p}<0.05$ vs basal.

medically treated patients with previous IGT had a further worsening in glucose tolerance, becoming diabetic.

Carbohydrate metabolism was more affected in the patients treated with oct-LAR as compared to those treated with lan-SR. In fact, a significant increase in fasting glucose levels (mean increase of $17 \pm 16 \%$, $p<0.05$ vs basal) and a significant decrease in insulin levels (mean reduction from basal of $41.6 \pm 54 \%$, $p<0.05)$ was observed only with oct-LAR (Table 2). QUICKI changed only in patients treated with oct- 
LAR ( $p<0.05$ vs basal), whereas HOMA-IR similarly decreased in both subgroups (both $p=N S$ vs basal). No differences between patients who normalized (5/15 with lan-SR and 5/12 with oct-LAR) and patients that did not normalize IGF-I levels were shown.

In 12 of the 27 patients who were evaluated after 24-month medical therapy, fasting glucose levels slightly increased from $4.9 \pm 1.0$ to $5.2 \pm 0.9$ after 6 months $(p=N S)$ and then to $5.8 \pm 1.1$ $\mathrm{mmol} / /$ after 24 months of treatment ( $p<0.005 \mathrm{vs}$ basal), while fasting insulin decreased from $17.3 \pm 12$ to $12.5 \pm 5(p=N S)$ and then to $6 \pm 4 \mathrm{mU} / \mathrm{I}$ $(p<0.01$ vs basal and 6 months), respectively. HOMA-IR and QUICKI significantly changed only after 24 months (both $p<0.05$ vs basal and 6 months) (Fig. 2). The number of patients that had HOMA-IR values above 3.1 decreased from 7 to 1 after 24 months ( $p<0.05$ vs basal and 6 months), while the number of patients with QUICKI values lower than 0.30 decreased from 3 to $0(p<0.05$ vs basal and 6 months).

\section{DISCUSSION}

The present study confirms that most of acromegalic patients are insulin-resistant, with higher HOMA-IR and lower QUICKI values in respect to those of healthy subjects $(2-5,25)$. The degree of insulin resistance is independent from the glucose tolerance status $(2,25)$ and from circulating levels of GH or IGF-I (30). Moreover, these results suggest that HOMA-IR and QUICKI analysis are useful simple methods to study insulin sensitivity in acromegalic patients, in agreement with previous studies in different populations of insulin-resistant patients $(11,17-21)$.

Most interestingly, 6-month treatment with lan-SR or oct-LAR causes clear improvement of insulin re-

Table 2 - Hormonal and biochemical data: comparison between 6 month-medical therapy with lan-SR and oct-LAR.

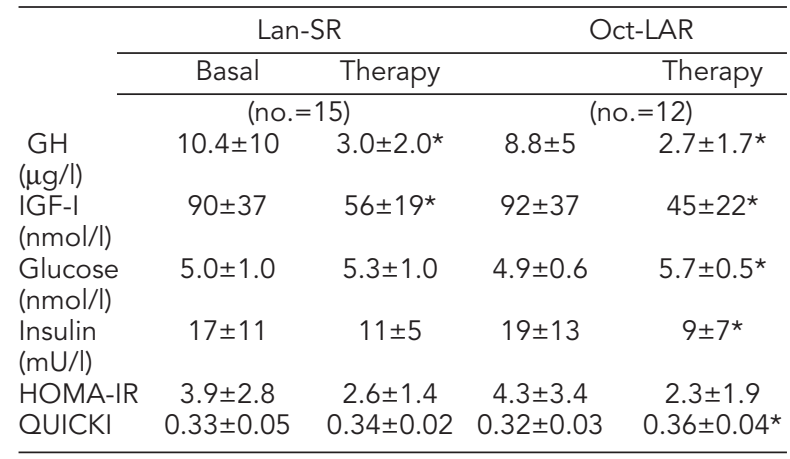

${ }^{*} p<0.05$ vs baseline.
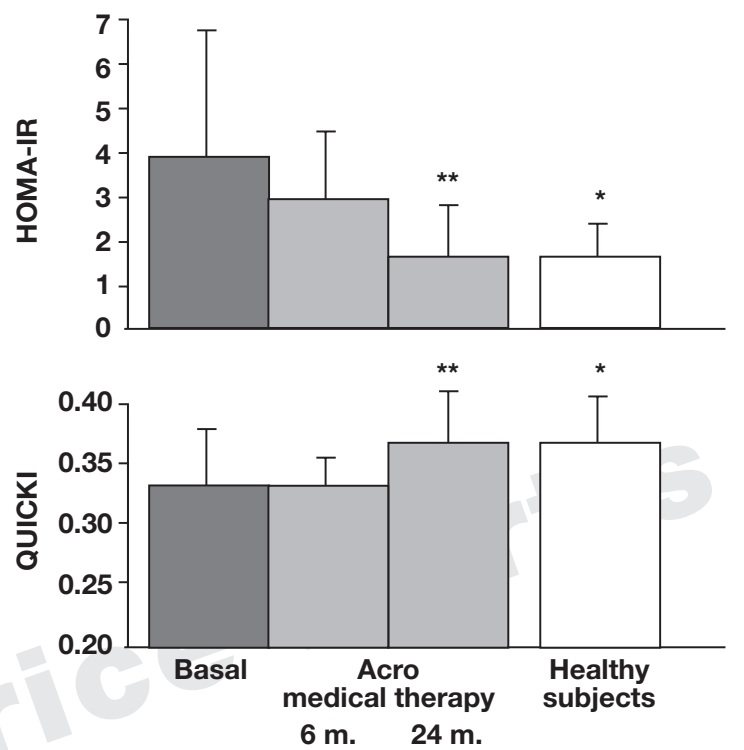

Fig. 2 - Insulin resistance by homeostasis model assessment (HOMA-IR) and insulin sensitivity by quantitative insulin check in$\operatorname{dex}(\mathrm{QUICKI})$ in 12 acromegalic patients before and after 6- or 24-month medical therapy and in 35 healthy subjects. ${ }^{*} \mathrm{p}<0.05$ vs basal, ${ }^{\star *} \mathrm{p}<0.05$ vs basal and 6 months.

sistance, as demonstrated by a significant decrease of HOMA-IR, associated with an increase of fasting glucose and a deep decrease of fasting insulin levels, as expected by the inhibition of insulin secretion secondary to somatostatin analogue administration. On the contrary, no significant change of QUICKI values was found. It is worth noting that 2 patients presented after 6-month therapy a worsening in glucose tolerance, becoming diabetics. These results are the balance between the somatostatin analogues inhibitory effects on pancreatic secretion of insulin and glucagon and the improvement of insulin resistance due to likely predominant GH decrease. No differences were noted either between the effects of different doses of the 2 drugs or between those patients who normalized $\mathrm{GH}$ levels and those who did not. These data confirm previous findings, obtained by euglycemic clamp, on the evaluation of insulin sensitivity improvement during sc daily administration of octreotide (27). Despite the decrease of insulin resistance found in most patients treated with analogues, a complete normalization of both HOMA-IR and QUICKI was observed only in patients successfully treated by pituitary surgery, as previously described (25).

Long-term treatment (24 months) seems to amplify the inhibition of pancreatic insulin secretion, so that also QUICKI values, besides insulin and 
HOMA-IR, significantly improved, despite a progressive increase in fasting glucose levels. These results indicate that long-acting somatostatin analogues have a continuous and progressive inhibitory action on pancreatic insulin secretion. These findings are in partial contrast to previous observations suggesting a diminished insulin inhibition with Tachiphilaxis (31). However they are concordant with more recent studies showing prolonged inhibitory effects on insulin secretion of long-term treatment with both octreotide (28) and lan-SR (26). Moreover, these properties may have opposite effects on final glucose homeostasis of medically treated acromegalic patients. Oct-LAR therapy was more powerful in decreasing IGF-I levels as well as in inhibiting insulin secretion, in respect to lan-SR, thus resulting in a higher impairment of glucose metabolism. These findings confirm previous data on comparison between the effects of lan-SR and oct-LAR (32) and suggest the importance of the evaluation of glucose metabolism before choosing the best first medical treatment approach and during the follow-up.

In conclusion, acromegalic patients have a high degree of insulin resistance that progressively improves during long-term therapy with different somatostatin analogues. These variations may be adequately monitored by using HOMA-IR and especially QUICKI.

\section{ACKNOWLEDGMENTS}

The Authors are grateful to Mrs. Deriu Rita for nursing help. The present study was partially supported by research grants 9906153187-00 from Ministero dell'Università and Ricerca Scientifica (MURST, Rome) and from Ospedale Maggiore IRCCS (Milan).

\section{REFERENCES}

1. Melmed S. Acromegaly. N. Engl. J. Med. 1990, 322: 966-977.

2. Foss M.C., Saad M.J.A., Paccola G.M.G.F., Paula F.J.A., Piccinato C.E., Moreira A.C. Peripheral glucose metabolism in acromegaly. J. Clin. Endocrinol. Metab. 1991, 72: 10481053

3. Moller N., Schmitz O., Jorgensen J.O.L., et al. Basal and insulin-stimulated substrate metabolism in patients with active acromegaly before and after adenomectomy. J. Clin. Endocrinol. Metab. 1992, 74: 1012-1019.

4. Muggeo M., Robert S.B., Roth J., Kahn R. The insulin resistance of acromegaly: evidence for two alterations in the insulin receptor on circulating monocytes. J. Clin. Endocrinol. Metab. 1979, 48: 17-25.

5. Hansen I., Tsalikian E., Beaufrere B., Gerich J., Haymond M., Rizza R. Insulin resistance in acromegaly: defects in both hepatic and extrahepatic insulin action. Am. J. Physiol. 1986, 250: E269-E273.
6. Butler P., Kryshak E., Rizza R. Mechanism of growth hormone-induced postprandial carbohydrate intolerance in human. Am. J. Phisiol. 1991, 260: E513.

7. Randle P., Garland P., Hales C., Newsholme E. The glucose fatty-acid cycle: its role in insulin sensitivity and the metabolic disturbances of diabetes mellitus. Lancet 1963, 1: 785-789.

8. Matthews D.R., Hosker J.P., Rudenski A.S., Naylor B.A., Treacher D.F., Turner R.C. Homeostasis model assessment: insulin resistance and $\beta$-cell function from fasting plasma glucose and insulin concentration in man. Diabetologia 1985, 28: 412-419.

9. The Mexico City Diabetes Study. A prospective analysis of the HOMA model. Diabetes Care 1996, 19: 1138-1143.

10. Haffner S.M., Miettinen H., Stern M.P. The Homeostasis model in the San Antonio Heart Study. Diabetes Care 1997, 20: 1087-1092.

11. Katz A., Nambi S.S., Mather K., et al. Quantitative insulin sensitivity check index: a simple, accurate method for assessing insulin sensitivity in humans. J. Clin. Endocrinol. Metab. 2000, 85: 2402-2410.

12. Bonora E., Targher G., Alberiche M., et al. Homeostasis model assessment closely mirrors the glucose clamp technique in the assessment of insulin sensitivity: studies in subjects with various degrees of glucose tolerance and insulin sensitivity. Diabetes Care 2000, 23: 57-63.

13. Ikeda Y., Suehiro T., Nakamura T., Kumon Y., Hashimoto K. Clinical significance of the insulin resistance index as assessed by homeostasis model assessment. Endocr. J. 2001, 48: 81-86.

14. Bastard J.P., Robert J.J., Jardel C., Buckert E., Grimaldi A., Hainque I. Is quantitative insulin sensitivity check index, a fair insulin sensitivity in humans? Diabetes Metab. 2001, 27: 69-70.

15. De Fronzo R.A., Tobin J.D., Andres R. Glucose clamp technique: a method for quantifying insulin secretion and resistance. Am. J. Phisiol. 1979, 237: 214-223.

16. Mc Auley K.A., Williams S.M., Mann J.I., et al. Diagnosing insulin resistance in the general population. Diabetes Care 2001, 24: 460-464.

17. Hermans M.P., Levy J.C., Morris R.J., Turner R.C. Comparison of insulin sensitivity tests across a range of glucose tolerance from normal to diabetes. Diabetologia 1999, 42: 678-687

18. Katsuki A., Sumida Y., Gabazza E.C., et al. Homeostasis model assessment is a reliable indicator of insulin resistance during follow-up of patients with type 2 diabetes. Diabetes Care 2001, 24: 362-365.

19. Matsumoto K., Miyake S., Yano M., et al. Glucose tolerance, insulin secretion, and insulin sensitivity in nonobese and obese Japanese subjects. Diabetes Care 1997, 20: 1562-1568

20. Lansang M.C., Williams G.H., Carroll J.S. Correlation between the glucose clamp technique and the homeostasis model assessment in hypertension. Am. J. Hypertens. 2001, 14: 51-53. 
21. Yildiz B.O., Gedik O. Insulin resistance in polycystic ovarian sindrome: hyperandrogenemia versus normoandrogenemia Eur. J. Obstet. Gynecol. Reprod. Biol. 2001, 100: 62-66.

22. Bonora E., Formentini G., Calcaterra F., et al. HOMA-estimated insulin resistance is an independent predictor of cardiovascular disease in type 2 diabetic subjects. Diabetes Care 2002, 25: 1135-1141.

23. Hanley A.J.G., Williams K., Stern M.P., Haffner S.M. Homeostasis model assessment of insulin resistance in relation to the incidence of cardiovascular disease. Diabetes Care 2002, 25: 1177-1184.

24. Quon M.J. Editorial: Limitation of the fasting glucose to insulin ratio as an index of insulin sensitivity. J. Clin. Endocrinol. Metab. 2001, 86: 4615-4617.

25. Kasayama S., Otsuki M., Takagi M., et al. Impaired $\beta$-cell function in the presence of reduced insulin sensitivity determines glucose tolerance status in acromegalic patients. Clin. Endocrinol. (Oxf.) 2000, 52: 549-555.

26. Giusti M., Gussoni G., Cuttica C.M., Giordano G. Effectiveness and tolerability of slow release lanreotide treatment in active acromegaly: six months report on an Italian multicenter study. J. Clin. Endocrinol. Metab. 1996, 81: 2089-2097.
27. Ho K.K.Y., Jenkins A.B., Furler S.M., Borkman M., Chisholm D.J. Impact of octreotide, long-acting somatostatin analogue, on glucose tolerance and insulin sensitivity in acromegaly. Clin. Endocrinol. (Oxf.) 1992, 36: 271-279.

28. Arosio M., Macchelli S., Rossi C.M., Casati G., Biella O., Faglia G. Effects of treatment with octreotide in acromegalic patients - a multicenter Italian study. Eur. J. Endocrinol. 1995, 133: 922-928.

29. Colao A., Ferone D., Marzullo P., et al. Long-term effects of depot long acting somatostatin analogue octreotide on hormone levels and tumor mass in acromegaly. J. Clin. Endocrinol. Metab. 2001, 86: 2779-2796.

30. Luger A., Prager R., Gaube S., Graf H., Klauser R., Schertnthaner G. Decreased peripheral insulin sensitivity in acromegalic patients. Exp. Clin. Endocrinol. 1990, 95: 339-343.

31. Lamberts S.W., Uitterlinden P., Verschoor L., Van Dogen K.J., Del Pozo E. Long-term treatment of acromegaly with the somatostatin analogue SMS 201-995. N. Engl. J. Med. 1985, 313: 1576-1580.

32. Ronchi C., Epaminonda P., Cappiello V., Beck-Peccoz P., Arosio M. Effects of two different somatostatin analogues on glucose metabolism and $\mathrm{GH}$ levels in acromegaly. J. Endocrinol. Invest. 2002, 25: 502-507. 